\title{
Повышение точности определения компенсации примесей в чистом, слабокомпенсированном германии по величине поля пробоя
}

\author{
(C) В.Ф. Банная \\ Московский педагогический государственный университет (МПгУ), \\ 119991 Москва, Россия \\ E-mail: enikitina@sci.pfu.edu.ru
}

(Получена 10 мая 2016 г. Принята к печати 16 мая 2016 г.)

Показано, что измерение поля электрического пробоя $E_{\mathrm{np}}$ в классически сильном магнитном поле $(H)$ при $T=4.2 \mathrm{~K}$ позволяет определять величину степени компенсации $K$ в чистом германии с $K<50 \%$ значительно точнее, чем при $H=0$. Введен параметр $S=E_{\mathrm{np}} / H$ и рассчитана его зависимость $S=f(K)$, полученная кривая позволяет определить $K$, если известны $H$ и $E_{\text {nр. }}$ Для уменьшения сопротивления образцов рекомендуется проводить измерения при наличии примесно́й подсветки. Показано, что при малых интенсивностях такого возбуждения величина $E_{\mathrm{np}}$ не меняется.

DOI: $10.21883 /$ FTP.2017.03.44197.8315

Зависимость поля низкотемпературного примесного пробоя $\left(E_{\mathrm{np}}\right)$ в чистом германии от степени компенсации примесей $(K)$ подробно изучена в работах $[1-4]$. Показано, что в этих условиях $(T=4.2 \mathrm{~K}$, $N_{a}+N_{d} \lesssim 5 \cdot 10^{14} \mathrm{~cm}^{-3}$, отсутствие подсветки) $E_{\mathrm{np}}$ зависит от $K\left(E_{\mathrm{np}}=f(K)\right)$. В этих работах даны теоретические обоснования и представлены соответствующие зависимости для $\mathrm{Ge} n$ - и $p$-типов, проверенные на большом числе образцов. Эти зависимости лежат в основе экспресс-метода определения $K=N_{a} / N_{d}$ (для $n$-типа проводимости), где $N_{a}$ - концентрация акцепторов, $N_{d}$ - концентрация доноров.

Однако чувствительность этого метода, а следовательно, и точность определения $K$ неодинакова для слабои сильнокомпенсированных образцов. Назовем область компенсаций с $K \lesssim 50 \%$ слабой, а с $K>50 \%$ сильной. Это связано с существенно разной крутизной зависимости $E_{\text {np }}(K)$ в этих областях. Так, при изменении $K$ от $\sim 10$ до $50 \% E_{\mathrm{np}}$ меняется от $\sim 3 \mathrm{~B} / \mathrm{cm}$ до $4.5 \mathrm{~B} / \mathrm{cm}$ в $n$-Ge и от $\sim 2$ до $3.5 \mathrm{~B} / \mathrm{cm}$ в $p$-Ge. Для сравнения, в интервале компенсаций от 50 до 90\% эти изменения составляют: от 4.5 до $10.8 \mathrm{~B} / \mathrm{cm}$ в $n$-Ge и от 3.5 до $7.5 \mathrm{~B} / \mathrm{cm}$ в $p$-Ge. (Данные получены из обработки результатов работ $[2,3])$.

При компенсациях $K>90 \%$ наблюдается так называемое затягивание $E_{\mathrm{np}}$, обусловленное „включением“ механизма рассеяния на оптических фононах [4].

Точность определения степени компенсации примесей в чистом Ge из кривых $E_{\mathrm{np}}=f(K)$ можно значительно повысить, проводя измерения проводимости образцов $\sigma=f(E)$ в магнитном поле. Подробные исследования влияния магнитного поля $(H)$ на электрический пробой в $n$ - и $p$-Ge при $T=4.2 \mathrm{~K}$ представлены в работе [5]. Показано, что в области классически сильных магнитных полей с ростом $H$ наблюдается возрастание $E_{\mathrm{np}}$. Это связано с тем, что поперечное магнитное поле „охлаждает“ носители заряда, уменьшая их среднюю энергию, что приводит к уменьшению вероятности ударной ионизации и как следствие к увеличению $E_{\mathrm{np}}$. Зависимость $E_{\mathrm{np}}(H)$ носит линейный характер, так как в выражениях для вероятностей ударной ионизации и термической рекомбинации эти поля входят в комбинации $E_{\mathrm{np}} / H$, причем с ростом $K$ это отношение растет [5]. Этот факт и позволяет повысить точность определения $K$, исходя из увеличения крутизны зависимости $E_{\mathrm{np}}(K)$, измеренной в магнитном поле.

На рис. 1 представлены кривые $E_{\mathrm{np}}(K)$, полученные при $H=11600$ э. Для сравнения приведены аналогичные зависимости для $H=0$. (Все расчеты выполнены по данным работы [5]).

Видно, что в указанном магнитном поле, в интервале компенсаций $10-50 \% E_{\mathrm{np}}$ меняется от $\sim 40$ до $70 \mathrm{~B} / \mathrm{cm}$ в $p$-Gе и от $\sim 36$ до $60 \mathrm{~B} / \mathrm{cm}$ в $n$-Ge.

Чтобы учесть влияние $H$ введем параметр $S=E_{\mathrm{np}} / H$, который также зависит от величины $K$. На рис. 2 представлены кривые $S=f(K)$ для $n$ - и $p$-Ge при $K \leq 50 \%$, рассчитанные по данным работы [5] (точки экспериментальные данные). Видно, что, зная величину

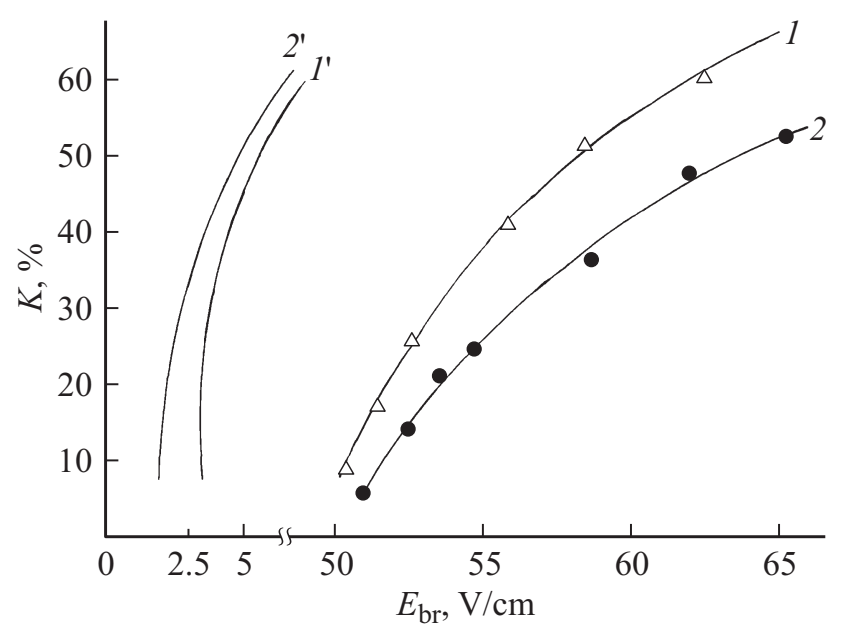

Рис. 1. Зависимость $E_{\mathrm{np}}(K)$ для чистых образцов $\mathrm{Ge}$ при $T=4.2 \mathrm{~K}$ и термовозбуждении: $1-n$-Ge, $2-p$-Ge, $H=11600$ э; $1^{\prime}-n$-Ge, $2^{\prime}-p$-Ge, $H=0$. 


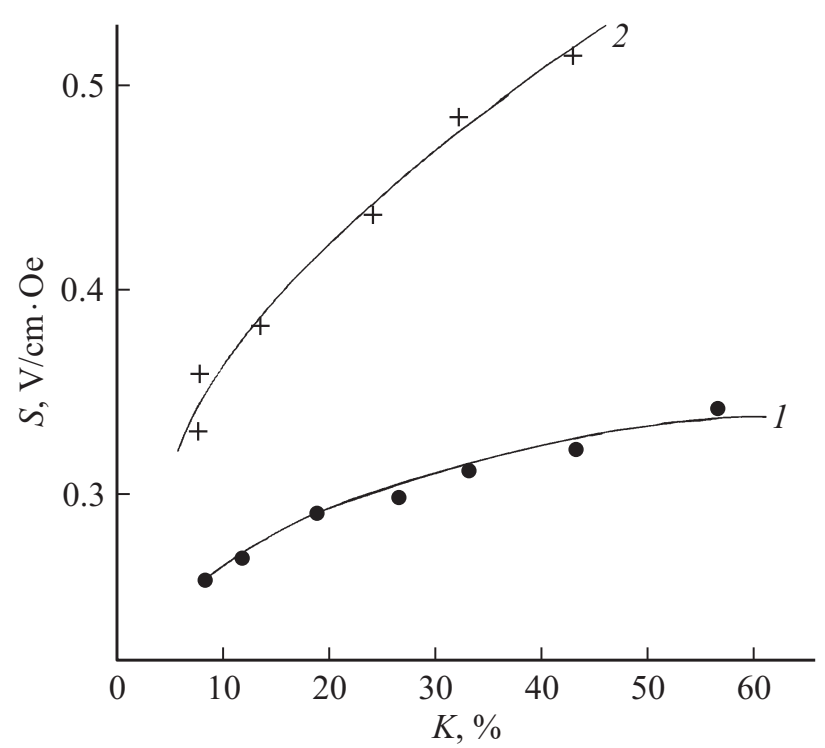

Рис. 2. Зависимость параметра крутизны $S=f(K)$ для $n$-Ge (кривая 1) и $p$-Ge (кривая 2).

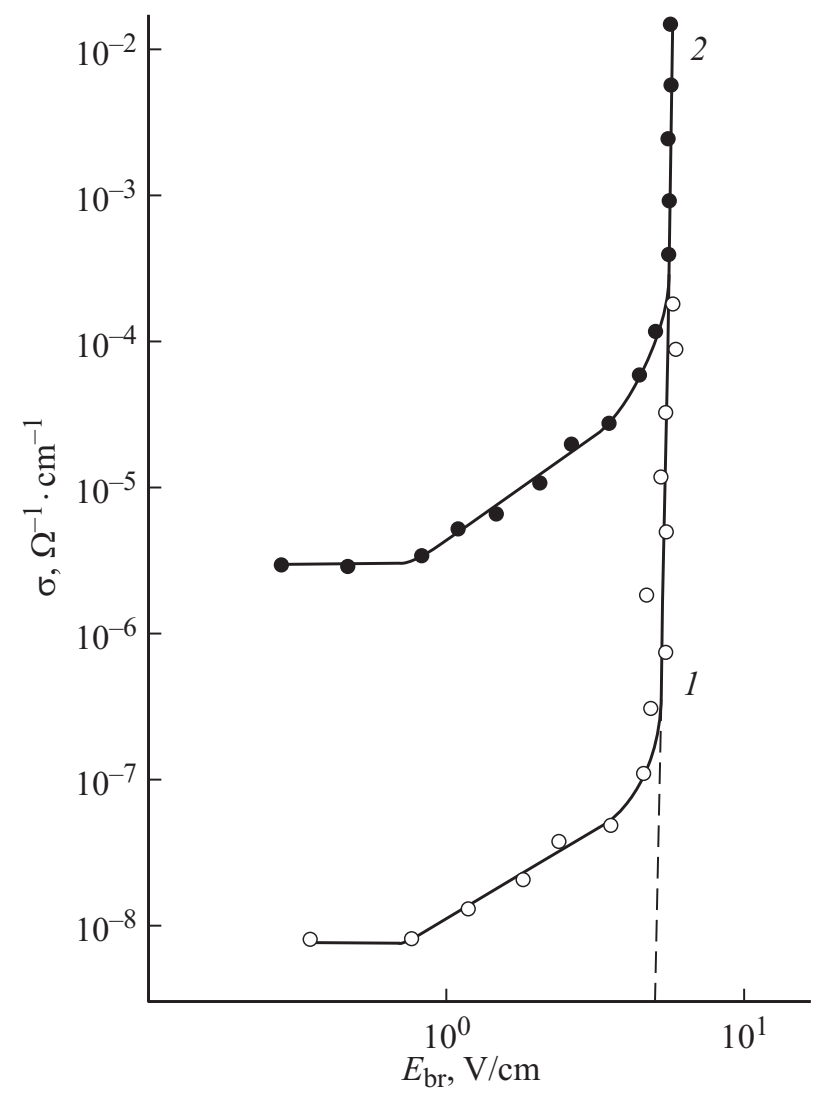

Рис. 3. Зависимость $\sigma(E)$ для $n$-Ge при $T=4.2 \mathrm{~K}: 1-$ в темновых условиях; $2-$ при фоновой подсветке $(T=300 \mathrm{~K})$.

магнитного поля, в котором проводятся измерения $E_{\mathrm{np}}$ и зависимость $S(K)$, можно определить $K$ в случае слабокомпенсированных образцов значительно точнее, чем при $H=0$.
Гальваномагнитные измерения чистого $\mathrm{Ge}$ при $T=4.2 \mathrm{~K}$ в условиях только термогенерации сопряжены с трудностями, обусловленными большим сопротивлением образцов (удельное сопротивление $(\rho)$ образцов чистого Ge при гелиевых температурах $\left.\rho \approx 10^{9} \mathrm{OM} \cdot \mathrm{cm}\right)$. Включение магнитного поля приводит к дополнительному увеличению $\rho$, так как Ge обладает большим магнитосопротивлением. Ситуацию можно значительно упростить, уменьшить сопротивление образцов, проводя измерения в условиях примесной подсветки (например, фонового при $T=300 \mathrm{~K}$ ).

Возникает вопрос - будет ли примесное фотовозбуждение влиять на величину $E_{\mathrm{np}}$. На рис. 3 представлены типичные зависимости $\sigma(E)$ для образцов $n$-Ge, измеренные в темновых условиях и при наличии фоновой подсветки $(\sigma-$ удельная электропроводность образцов).

Видно, что наличие подсветки приводит к возрастанию $\sigma$ больше чем в 100 раз, однако $E_{\mathrm{np}}$ в пределах точности измерений в обоих случаях совпадает.

Фоновая подсветка вызывает появление в зоне проводимости неравновесных носителей в результате ионизации примесей излучением. Полная концентрация носителей равна сумме концентраций равновесной и неравновесной. Увеличение концентрации неравновесных носителей в зоне приводит к накоплению электронов в возбужденных состояниях примесных центров $n_{i}$. При достаточно больших $n_{i}$ следует ожидать уменьшение величины $E_{\text {nр }}$, так как основным процессом может стать ионизация возбужденных состояний. Однако при не слишком больших интенсивностях подсветки величина $n_{b}$ близка к числу электронов в зоне, т. е. в этом случае сохраняется ситуация, когда $\left(n+n_{i}\right) \ll N_{a}, N_{d}-N_{a}$, соответствующая темновому случаю. Таким образом, при не слишком больших уровнях генерации фоновый подсвет не приводит к изменению величины $E_{\text {np }}$.

Таким образом, проводя измерение $E_{\mathrm{np}}$ при не слишком больших уровнях примесной подсветки в классически сильных магнитных полях при $T=4.2 \mathrm{~K}$ и используя зависимость $S(K)$, можно значительно повысить точность определения $K$ в слабокомпенсированном чистом $n$ - и $p$-Ge.

\section{Список литературы}

[1] В.Ф. Банная, Л.И. Веселова, Е.М. Гершензон, В.Р. Гринберг. ФТП, 5, 155 (1971).

[2] В.Ф. Банная, Л.И. Веселова, Е.М. Гершензон, В.А. Чуенков. ФТП, 7, 1972 (1973).

[3] В.Ф. Банная, Л.И. Веселова, Е.М. Гершензон, Ю.А. Гурвич. ФТП, 10, 452 (1976).

[4] В.Ф. Банная, Е.М. Гершензон, Л.И. Веселова. ФТП, 13, 46 (1979).

[5] В.Ф. Банная, Л.И. Веселова, Е.М. Гершензон, В.А. Чуенков. ФТП, 10, 338 (1976).

Редактор А.Н. Смирнов 


\title{
Improving the accuracy of determination \\ of compensation of impurites in pure \\ weakly compensated germanium \\ largest breakdown field
}

\section{V.F. Bannaya}

Moscow State University of Education (MSPU),

119991 Moscow, Russia

\begin{abstract}
The article shows that the measurement field of electric breakdown $E_{\mathrm{np}}$ of the Insulation in a classically strong magnetic field $(H)$ when $T=4.2 \mathrm{~K}$ allows to determine the value of the degree of compensation To net in germanium $K<50 \%$ is much more accurate than when $H=0$. Parameter $S=E_{\mathrm{np}} / H$ the dependence of $S=f(K)$ is introduced calculated; the curve allows to determine $K$, if $H$ and $E_{\mathrm{np}}$ is known. To reduce the resistance of the samples it recommended to carry out measurements in the presence of the impurity illumination. It is shown that at low excitation intensities of such magnitude $E_{\mathrm{np}}$ does not change.
\end{abstract}

